\title{
Improved Specificity and False Discovery Rates for Multiplex Analysis of Changes in Strain-Specific Anti-Influenza IgG
}

\author{
Dongmei Li $\mathbb{D},{ }^{1}$ Jiong Wang $\left(\mathbb{D},{ }^{2}\right.$ John J. Treanor, ${ }^{3}$ and Martin S. Zand $\mathbb{D}^{1,2,4}$ \\ ${ }^{1}$ Informatics Core, Clinical and Translational Science Institute, University of Rochester Medical Center, Rochester, NY, USA \\ ${ }^{2}$ Department of Medicine, Division of Nephrology, University of Rochester Medical Center, Rochester, NY, USA \\ ${ }^{3}$ Department of Medicine, Division of Infectious Diseases, University of Rochester Medical Center, Rochester, NY, USA \\ ${ }^{4}$ Rochester Center for Health Informatics, University of Rochester Medical Center, Rochester, NY, USA
}

Correspondence should be addressed to Dongmei Li; dongmei_li@urmc.rochester.edu and Martin S. Zand; martin_zand@urmc.rochester.edu

Dongmei Li and Jiong Wang contributed equally to this work.

Received 26 November 2018; Accepted 27 February 2019; Published 15 April 2019

Academic Editor: Rafik Karaman

Copyright (c) 2019 Dongmei Li et al. This is an open access article distributed under the Creative Commons Attribution License, which permits unrestricted use, distribution, and reproduction in any medium, provided the original work is properly cited.

We describe a statistical approach to compare absolute antibody concentrations, both within and across subjects, derived from a multidimensional measurement of IgG binding to the influenza surface receptor hemagglutinin (HA). This approach addresses a fundamental problem in the field of vaccine immunology: how to accurately compare the levels of antibodies against multiple influenza strains. The mPlex-Flu assay can simultaneously measure the concentration of IgG antibodies against up to 50 influenza strains with only $\leq 10 \mu l$ of serum. It yields mean fluorescence intensity (MFI) over a 4-log range with low inter- and intrasample variability. While comparison of IgG binding to a single HA between subjects is straightforward, variations in binding behavior across influenza strains, coupled with reagent variations, make quantifying and comparing binding between multiple HA subtypes within subjects challenging. In this paper, we first treat such HA variations as an independent antigen and calculate each subtype antibody concentration using its own standard curve, normalizing variations in HA binding. We applied this method to the analyses of data from an $\mathrm{H} 5$ influenza clinical vaccine study. The results demonstrated that there are differences in coefficient estimates and in results of "comparing groups" between those with versus those without consideration of subtype antibody variations. Then, we used simulation studies to show the importance of taking the subtype antibody variations into account in HA strain antibody data analysis. Using a common standard curve for all subtype antibodies resulted in both inflated type I error and lowered specificity when comparing different treatment groups. Our results suggest that using individual standard curves for each influenza HA strain, and independently calculating anti-HA IgG concentrations, allows for adjustment of influenza HA subtype variations in treatment group comparisons in clinical vaccine studies. This method facilitates the direct comparison of serum antiHA IgG concentrations against different influenza HA subtypes for multiplex assays.

\section{Introduction}

Estimating the concentration of antibodies directed against the major influenza viral surface protein hemagglutinin (HA) is critical for studies of antibody-mediated influenza immunity and especially for vaccine development [1]. Because the influenza virus mutates frequently, new strains are always emerging that can evade prior antiHA IgG-mediated immunity, necessitating new vaccine formulations each year. Recently, emphasis has been placed on creating vaccines that generate broadly crossreactive antibodies, protecting against many influenza strains [2]. Thus, the ability to simultaneously measure antibody binding against multiple influenza HA and to accurately compare antibody binding across many influenza strains, especially within and between subject binding distributions, is highly desirable. However, a major impediment to such comparisons is the variability of such multiple comparisons across many HA reagents, both for technical and statistical reasons. 
We have previously described a multiplex-based method that simultaneously measures antibody binding against up to 50 influenza strain hemagglutinin proteins, the mPlex-Flu assay $[3,4]$. HA proteins mediate viral attachment and entry into target cells [5]. Antibodies that bind to influenza HA can prevent or attenuate the severity of influenza infection. In mPlex-flu assay, each recombinant influenza strain HA couples to fluorescent microbeads; then, the mixtures of the HA-coated beads are used to simultaneously detect antibodies binding to multiple influenza strains. This multidimensional analytic method generates a continuous value for the mean fluorescence intensity (MFI), accurate over a 4-log range, reflecting antibody binding.

As a multidimensional assay, mPlex-flu assay is different from traditional titer-based assays such as the hemagglutinin inhibition (HAI) $[6,7]$ and microneutralization (MN) $[8,9]$ assays that measure IgG antibody binding to single-HA proteins. Importantly, this feature allows for the measurement of multidimensional cross-reactive immunity $[4,10]$, which is crucial when assessing whether a vaccine will provide broad protection against many influenza strains. This assay provides accurate concentrations of anti-HA IgG against different influenza strains and is able to detect statistically significant variations between experimental groups in clinical vaccine studies, compared to the HAI and MN assays.

Translating MFI measured by multiplex assay into absolute concentrations of anti-HA antibodies creates unique challenges compared to standard monoplex semiquantitative assays (e.g., ELISA, HAI). First, mPlex-Flu assay uses influenza strain-specific rHA coupling microbeads to detect the anti-HA antibodies. However, traditional quantitative assays (e.g., ELISA [11], Luminex assay [12]) use immunoglobulinspecific capture antibody to couple microbeads to estimate the antibodies concentrations. Second, between-strain differences in HA molecular properties can cause slight variations in the density of the different HA's coating multiplex beads, resulting in slightly altered HA saturation and IgG binding characteristics [3, 4] (Figure 1). In addition, the assay is used to measure binding of a mixture of antibodies in sera that bind to multiple different sites on the HA protein, each with different affinities. The assessed antibody responses are polyclonal, but creating a precise mixture of monoclonal antibodies, targeting 20-40 different subtypes of influenza HA protein for 40-50 different $\mathrm{HA}$, is technically unfeasible. Thus, a mixture of polyclonal sera with reactivities against all HA strains must be used. Finally, traditional statistical methods for analyzing concentration data $[11,13]$, using one common standard curve for all subtypic HAs [12], do not account for reagent binding differences between captured proteins. This may lead to increased Type I statistical errors and lowered specificity, when comparing treatment groups in clinical vaccine studies.

To address these issues, to accurately calculate the concentration unit of influenza virus HA-specific antibodies, and to normalize the differences between subtype strains, we calculated individual standard curves for each recombinant HA coupled bead set. Using this method, we derived the absolute anti-HA antibody concentration for each influenza strain using a five-parameter logistic regression model. Importantly, we estimated strain-specific parameters for each of the different
HA subtype strains in the assay. We then applied this method to data from mPlex-Flu analysis of treatment groups of an influenza vaccine clinical trial (DMID 08-0059 [14]).

The results demonstrated differences in longitudinal estimates and comparisons from linear mixed effects models when comparing different treatment groups. Simulation studies showed that taking HA subtype variations into account in influenza anti-HA multiplex vaccination analysis lowered false discovery rates (FDR) and improved the specificity of the comparisons between treatment groups. Establishing individual standard curves for each influenza virus HA subtype will be extremely useful for the development of broadly crossreactive influenza vaccines. This method is generalizable to multiplex assays of polyclonal, antibody-mediated immunity against viruses with significant strain variation.

\section{Methods}

2.1. Human Subjects Ethics Statement. This study was approved by the Research Subjects Review Board at the University of Rochester Medical Center (RSRB approval number RSRB00012232). The clinical samples were analyzed under secondary use consent, and written informed consent was obtained from all participants and kept on file per RSRB regulations. Research data were coded such that subjects could not be identified, directly or through linked identifiers, in compliance with the Department of Health and Human Services Regulations for the Protection of Human Subjects (45 CFR 46.101(b) [14]. Subject identification numbers were reencoded for publication.

\subsection{Vaccine Study Design and Sample Collection. Data and} serum samples used in this report were obtained from stored samples generated by a prospective clinical trial of $\mathrm{H} 5$ influenza vaccination (DMID 08-0059) [14]. Briefly, 64 previously $\mathrm{H} 5$ influenza-vaccinated (PR) and 30 healthy adults not previously vaccinated against $\mathrm{H} 5$ influenza strains (UP) were vaccinated with an intramuscular inactivated $\mathrm{A} /$ Indonesia/5/05 (A/Ind05) vaccine in two doses (Figure 2). The antibody concentration data from the different doses within the same vaccine treatment group were adjusted in the statistical analysis using linear mixed effects models [15]. All of the $\mathrm{PR}$ group received the intramuscular inactivated A/Vietnam/1203/04 (A/Vie04) vaccine in 2005-2006. Of these subjects, 16 had received a vaccine containing the $\mathrm{rHA}$ of A/Hong Kong/156/97(A/HK97) in 1997-1998 and are designated as the multiply primed group (MPR). Subjects of $\mathrm{PR}$ and MPR groups were administrated single doses of the A/Ind05 influenza vaccine. Subjects in MPR group received 2 identical vaccinations separated by 28 days. Serum samples were collected before vaccination (Day0) and on days 3,7 , $14,28,56$, and 180 after vaccination. Serum samples were also collected from the UPR group on days $3,7,14$, and 28 days after the second immunization.

2.3. mPlex-Flu Analysis. We estimated concentrations of IgG antibodies against $45 \mathrm{HA}$ strains of influenza viruses in the serum samples from the DMID 08-0059 vaccine study using 


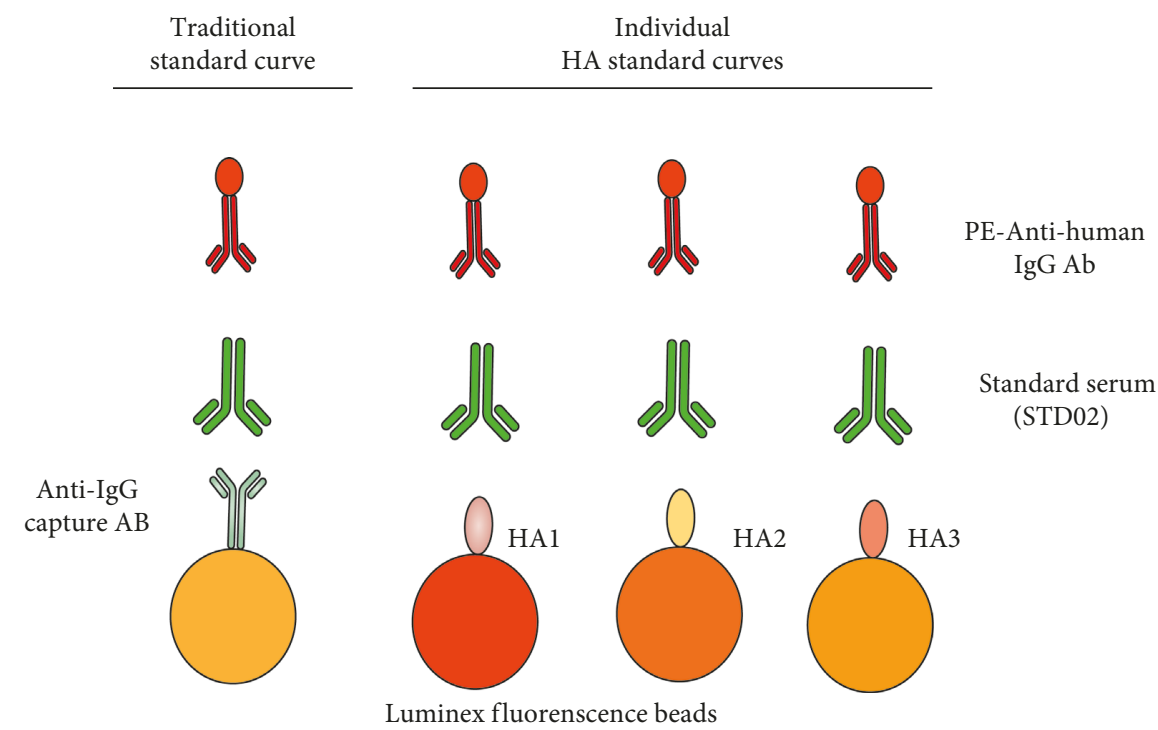

Figure 1: Principal of the mPLEX-Flu assay standard curve generation.

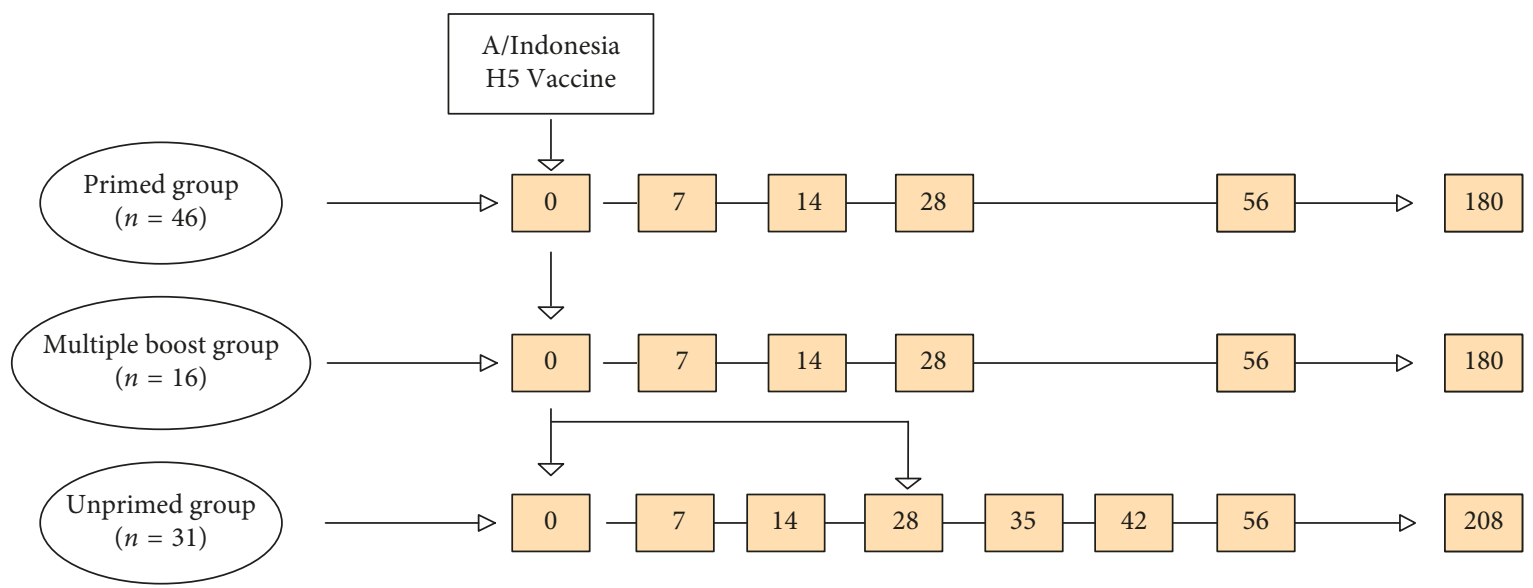

FIgURE 2: Study design of the prospective clinical trial of H5 influenza vaccination (DMID 08-0059) with an intramuscular inactivated A/ Indonesia/5/05 (A/Ind05) H5 influenza vaccine. The inactivated A/Indonesia/5/05 (A/Ind05) intramuscular influenza vaccine was used to vaccinate all subjects in the three cohorts. The inactivated subvirion influenza A/Vietnam/1203/04 (A/Vie04) vaccine in 2005-2006 was used to vaccinate the primed and the multiple boost groups. The baculovirus expressed recombinant influenza A/Hong Kong/156/97 vaccine (A/ HK97) in 1997-1998 was also used to vaccinate the multiple primed group. The unprimed group was vaccinated the A/Ind05 vaccine and a second booster vaccine at 28 days. Blood samples were collected as shown in the orange blocks: before vaccination (Day 0$)$ and on days 7,14 , 28,56 , and 180 after vaccination. For the unprimed group, blood samples were collected before vaccination (Day 0 ) and on days 7,14 , and 28 before boosting on day 28 and then on days 7 (Day 35), 14 (Day 42), 28 (Day 56), and 180 (Day 208) after boosting.

the mPlex-Flu assay [3]. All recombinant HA (rHA) proteins were produced by our lab, with trimerization domain on the end of C-terminal, including the HA heads. All rHAs were in trimer structure. Briefly, a panel of rHAs coupled mPlex-Flu beads listed on Table 1 were mixed and incubated with $20 \mu \mathrm{l}$ of diluted human sera for 2 hours, at 500 beads per each bead's region in the 96-well filtration plates (Millipore, Billerica, MA) at $4^{\circ} \mathrm{C}$, on a rotary shaker (500 rpm) in the dark. The wells were washed twice and then incubated with 1:400 diluted PE conjugated anti-human IgG ( $\gamma$ chain specific) secondary antibodies (SouthernBiotech, AL) in the dark at room temperature for 2 hours with gentle agitation (500 RPM). After three additional washes, the beads in each well were suspended in Luminex Magpix Drive Fluid (Luminex, Austin, TX) and analyzed on a MagPix multiplex reader (Luminex, Austin, TX), and the results obtained were measured in median fluorescence intensity (MFI).

2.4. Standard Serum and Standard Curves of mPlex-Flu Assay. Positive control serum for the seasonal influenza virus mPlexFlu (STD02) was created by pooling four positive sera from the subjects who had confirmed high concentrations of IgG antibodies against all $\mathrm{H1}, \mathrm{H} 3$ seasonal influenza virus strains and most $\mathrm{H} 5$ avian influenza virus strains. The total IgG concentration of this serum was $9.07 \mathrm{mg} / \mathrm{ml}$, as estimated by ELISA using a purified IgG standard (Abcam INC, MA, USA). Using this control serum, the traditional standard curve of 
TABLe 1: The mPlex-Flu assay panel of seasonal influenza viruses, H5 clades, and subclades.

\begin{tabular}{|c|c|c|c|c|}
\hline Influenza virus type & Subtypes & Full name of viruses & Abbreviation & H5 clades/subclades \\
\hline \multirow{37}{*}{ A } & \multirow{6}{*}{$\mathrm{H} 1$} & A/South Carolina/1/18 & A/SC18 & \\
\hline & & A/Puerto Rico/8/1934 & A/PR8 & \\
\hline & & A/USSR/90/1977 & A/USSR77 & \\
\hline & & A/New Caledonia/20/1999 & A/NewCall99 & \\
\hline & & A/Texas/36/1991 & A/Tex91 & \\
\hline & & A/California/07/2009 & A/Cali09 & \\
\hline & \multirow[t]{3}{*}{$\mathrm{H} 2$} & A/Japan/305/1957 & A/Jap57 & \\
\hline & & A/Port Chalmers/1/1973 & A/PC73 & \\
\hline & & A/Hong Kong/1/1968 & A/HK68 & \\
\hline & \multirow[t]{9}{*}{$\mathrm{H} 3$} & A/Perth/16/2009 & A/Per09 & \\
\hline & & A/Victoria/361/2011 & A/Vic11 & \\
\hline & & A/Texas/50/2012 & $\mathrm{A} / \mathrm{Tex} 12$ & \\
\hline & & A/Hong Kong/156/97 & $\mathrm{A} / \mathrm{HK} 97$ & 0 \\
\hline & & A/Viet Nam/1203/2004 & A/Viet04 & 1 \\
\hline & & A/Cambodia/P0322095/2005 & A/Cam05 & 1.1 \\
\hline & & $\mathrm{A} /$ Indonesia/5/05 & A/Ind05 & 2.1.3.2 \\
\hline & & A/Turkey/65596/2006 & A/TK06 & 2.2 .1 \\
\hline & & A/Common Magpie/Hong Kong/5052/2007 & $\mathrm{A} / \mathrm{cmHK} 07$ & 2.3.2.1 \\
\hline & \multirow{15}{*}{ H5 } & $\mathrm{A} /$ Shenzhen $/ 406 \mathrm{H} / 2006$ & A/SZ06 & 2.3 .4 \\
\hline & & A/Chicken/Guangxi/12/2004 & A/chiGX04 & 2.4 \\
\hline & & A/Chicken/Korea/es/2003 & A/chiKR03 & 2.5 \\
\hline & & A/Silky Chicken/Hong Kong/SF189/01 & A/s.chiHK01 & 3 \\
\hline & & A/Goose/Guiyang/337/2006 & A/gooGY06 & 4 \\
\hline & & A/Duck/Guangxi/1378/2004 & A/ducGX04 & 5 \\
\hline & & A/Duck/Hubei/wg/2002 & A/ducHB02 & 6 \\
\hline & & A/Beijing/01/2003 & $\mathrm{A} / \mathrm{BJ} 03$ & 7.1 \\
\hline & & A/Chicken/Shanxi/2/2006 & A/chiSX06 & 7.2 \\
\hline & & A/Chicken/Henan/16/2004 & A/chiHN04 & 8 \\
\hline & & A/Goose/Shantou/1621/05 & A/gooST05 & 9 \\
\hline & & A/duck/Sichuan/NCXN10/2014(H5N1) & A/ducSC14 & 2.3.4.4 \\
\hline & & A/turkey/Washington/61-22/2014(H5N2) & A/turWash14 & 2.3.4.4 \\
\hline & & A/duck/Guangdong/wy11/2008(H5N5) & A/ducGD08 & 2.3.4.4 \\
\hline & & A/turkey/California/K1500169-1.2/2015(H5N8) & $\mathrm{A} /$ turCal15 & 2.3.4.4 \\
\hline & H6 & A/chicken/Taiwan/67/2013 & A/chTW13 & \\
\hline & \multirow{2}{*}{$\mathrm{H} 7$} & A/mallard/Netherlands/12/2000 & $\mathrm{A} / \mathrm{malNert} 00$ & \\
\hline & & A/rhea/North Carolina/39482/1993 & A/rheaNC93 & \\
\hline & H9 & A/guinea fowl/Hong Kong/WF10/1999 & A/gfHK99 & \\
\hline B & & $\mathrm{B} /$ Brisbane/60/2008 & B/Bris08 & \\
\hline \multirow{3}{*}{ HA domains } & & Head of A/Shanghai/1/2013 & H7 Head & \\
\hline & & Head of A/Indonesia/5/05 & H5 Head & \\
\hline & & Headof A/guinea fowl/Hong Kong/WF10/1999 & H9 head & \\
\hline \multirow{4}{*}{ Chimeric HA } & & \multirow{4}{*}{$\begin{array}{c}\text { cH5/1 (head of A/Ind05, stalk of A/PR8) } \\
\text { cH5/1 (head of A/Ind05, stalk of A/Cal09) } \\
\text { cH9/1 (head of A/gf/HK99, head of A/Cal09) } \\
\text { cH4/7 (Head of A/duck/Czech/1956, stalk of A/ } \\
\text { Shanghai/1/2013) }\end{array}$} & $\mathrm{cH} 5 / 1 \mathrm{PR}$ & \\
\hline & & & $\mathrm{cH} 5 / 1 \mathrm{Cal}$ & \\
\hline & & & $\mathrm{cH} 9 / 1$ & \\
\hline & & & $\mathrm{cH} 4 / 7$ & \\
\hline
\end{tabular}

Note. All antigens were trimeric.

total IgG concentrations was found using a goat-anti-human IgG Fc specific capture antibody (Sigma-Aldrich, MO, USA) coupled to Luminex beads. We assayed serial dilutions of STD02 serum to create the total IgG standard curve, beginning with an initial dilution of $1: 1000$, followed by serial four-fold dilutions and a blank control [3]. STD02 serum at the same dilutions was then used to generate individual standard curves for each of the 45 influenza strain subtypes [3].

2.5. Dose-Response Curve. The MFI-IgG concentration relationship was modeled using four-parameter and five- parameter logistic regression models [16, 17]. For the mPlex-Flu assay, we assume $y_{i s}$ is the response corresponding to dilution level $x_{i}$ for sth the strain subtype. Then $y_{i s}$ and $x_{i}$ are described by the nonlinear function

$$
y_{i s}=f\left(x_{i}, \theta_{s}\right)+\epsilon_{i s},
$$

where $\epsilon_{i s}$ follows normal distribution with mean $\mu=0$ and variance $\sigma^{2} g\left\{f\left(x_{i}, \theta_{s}\right), \tau\right\}$ where $g\left\{f\left(x_{i}, \theta_{s}\right), \tau\right\}$ is a function of $f\left(x_{i}, \theta_{s}\right)$. The functions $f\left(x_{i}, \theta_{s}\right)$ are different for fourparameter logistic regression models and five-parameter 
logistic regression models. The four-parameter logistic regression model $f\left(x_{i}, \theta_{s}\right)$ is given by

$$
f\left(x_{i}, \theta_{s}\right)=\theta_{2 s}+\frac{\theta_{3 s}-\theta_{2 s}}{1+\left(x_{i} / \theta_{4 s}\right)^{\theta_{1 s}}},
$$

while the five-parameter model $f\left(x_{i}, \theta_{s}\right)$ is

$$
f\left(x_{i}, \theta_{s}\right)=\theta_{2 s}+\frac{\theta_{3 s}-\theta_{2 s}}{\left(1+\left(x_{i} / \theta_{4 s}\right)^{\theta_{1 s}}\right)^{\theta_{5 s}}},
$$

where parameters for the $s^{\text {th }}$ strain subtype are denoted by $\theta_{2 s}$ for the minimum and $\theta_{3 s}$ for the maximum responses, $\theta_{4 s}$ is the concentration that results in $50 \%$ response, $\theta_{1 s}$ is the relative slope at the $50 \%$ response, and $\theta_{5 s}$ denotes the asymmetry in the dose-response relationship. When we use a common standard curve for all strain subtypes, the four-parameter and five-parameter logistic regression models will be given by

$$
\begin{aligned}
& f\left(x_{i}, \theta\right)=\theta_{2}+\frac{\theta_{3}-\theta_{2}}{1+\left(x_{i} / \theta_{4}\right)^{\theta_{1}}}, \\
& f\left(x_{i}, \theta\right)=\theta_{2}+\frac{\theta_{3}-\theta_{2}}{\left(1+\left(x_{i} / \theta_{4}\right)^{\theta_{1}}\right)^{\theta_{5}}},
\end{aligned}
$$

where all $\theta$ s will take the same value for all strain subtypes. Previous studies have found that the five-parameter logistic regression model is superior to the four-parameter model with respect to the accuracy of concentration estimates [18]. Thus, in our simulation studies, we used five-parameter logistic regression model to estimate the mean concentration.

\section{Results}

3.1. Standard Curve Generation for Each Strain Subtype. Traditional immunoassays (e.g., ELISA) generally fit one common five-parameter logistic regression model standard curve to all results of antibody binding to influenza HA strain subtypes. For example, when measuring human immune responses, an anti-human IgG capture antibody is often used to bind serial dilutions of IgG from a solution of known concentration, and a secondary indicator antibody is used to measure the mean fluorescence intensity (MFI) versus the IgG concentration for a standard curve $[11,13]$. The principle of assays is shown in Figure 1. However, we have found that using this same procedure to generate a single standard curve is problematic for the multiple assay (mPlex-Flu).

We found that the traditional standard curve (Figure 3(a)) generated from the IgG capture antibodies is different from the individual standard curves (Figures 3(b)-3(d) show three influenza strains as examples) generated from each specific influenza HA strain in mPlexFlu assay, shown as different parameters in the fitted fiveparameter logistic regression models. The traditional standard curve has parameters of $\left(\theta_{1}=5.44, \theta_{2}=9.68, \theta_{3}=\right.$ $\left.0.41, \theta_{4}=4.42, \theta_{5}=0.81\right)$ in the fitted five-parameter logistic regression model (Figure $3(\mathrm{a})$ ).

The multiple assay approach allowed us to fit a fiveparameter logistic regression model for each strain subtype with different parameters for each strain subtypes. For the three strain subtype examples, the estimated parameters in the fitted five-parameter logistic regression models were different as shown in Table 2 and Figures 3(b)-3(d). Table 2 also shows the SEM of those estimated parameters for the three strain subtypes. Therefore, we hypothesize that influenza strain subtype variations (e.g., sequence, density on multiplex bead surface, glycosylation, and charge) could account for or cause inaccuracy during subsequent immunoassay data analysis when comparing different treatment groups in clinical vaccine studies. In addition, with the influenza strain subtype variations being taken into account, it is likely that variations between the strains could be mathematically adjusted for at same time. The data after such adjustment should allow us compare the absolute concentration of IgG anti-influenza between subtype strains. Besides statistical methodology, one can also prepare Agspecific pools for each of the antigen. This can be done by affinity purification or calibration-free concentration analysis on a BiaCore SPR machine. This is a critical technical problem for the assessment of influenza antibody crossreactivity in most antibody response and vaccine studies.

3.2. Differences in Statistical Inferences with and without considering Strain Subtype Variations. In order to test our hypothesis, we compared the differences in antibody concentrations across three different vaccine treatment groups with data generated by mPlex-Flu assay. First, we used the linear mixed effects model to adjust IgG titres to 21 strains of H5 influenza viruses with the effects of age at enrollment, gender, ethnicity, dosages, and batches. Autoregressive 1 correlation structure was used in the linear mixed effects model to take into account the within-subject correlations [19]. Then, we compared the antibody concentration data against 21 strains of H5 influenza viruses using either (1) a traditional common standard curve used for all strain subtypes (Figure 3(a)) (without considering strain differences) or (2) an individual standard curve for all H5 strain subtypes to consider the strains' difference. The longitudinal concentration data in the logarithm were from three immunization treatment groups: unprimed (UP), primed (PR), and multiple primed (MPR). The data included $21 \mathrm{H} 5$ vaccine strain subtypes with 18606 total observations from 3 different groups, 2 different dosages ( $15 \mathrm{mcg}$ and $90 \mathrm{mcg}$ ), 5 different batches, 7 or 10 different days, and 93 different subjects. The concentration in the logarithm was checked to follow an approximately normal distribution; thus, linear mixed effects models were used to fit the log transformed concentration data to compare the three different vaccine groups with and without considering the $\mathrm{H} 5$ vaccine strain subtype variations $[20,21]$.

For the group comparisons, the Kenward-Roger method was used to estimate the standard error for fixed effects and the degrees of freedom for each parameter [22]. Restricted maximum likelihood estimators were used to obtain the parameter estimates in the linear mixed effects model. Figure 4 shows the longitudinal estimates of the three different vaccine treatment groups from linear mixed effects models with and without consideration of the H5 strain 


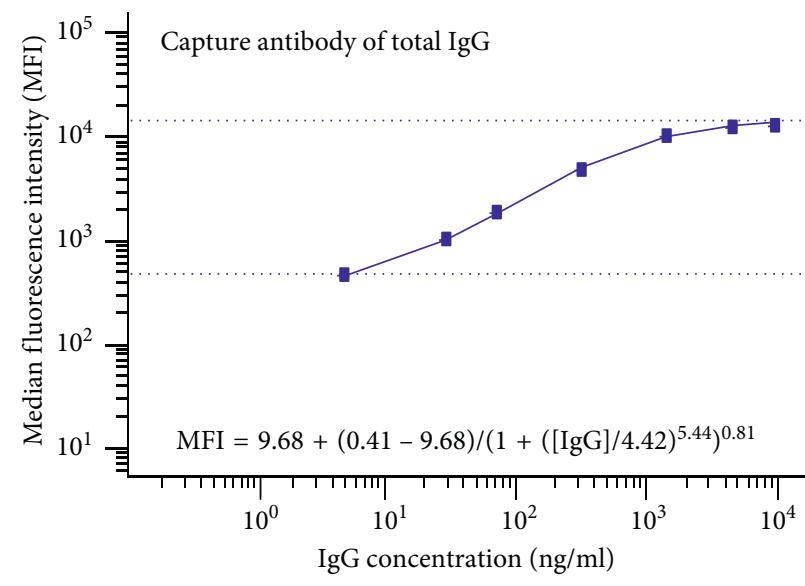

(a)

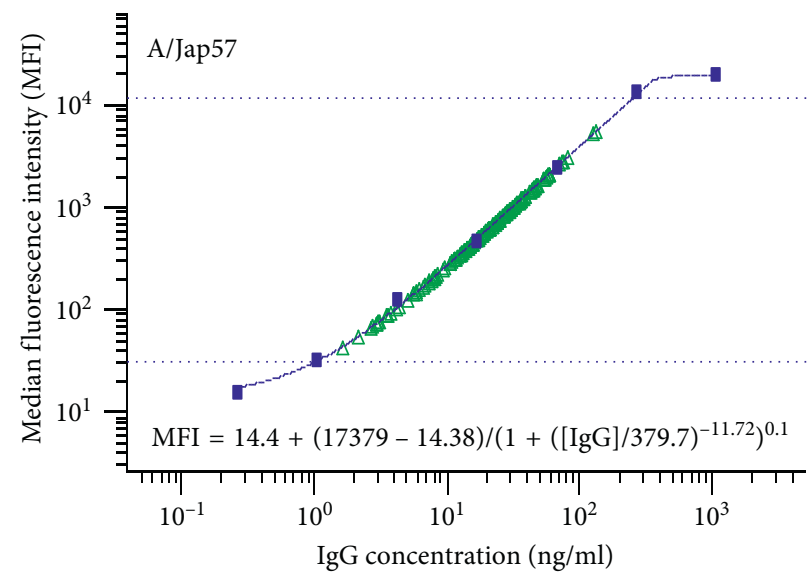

(c)

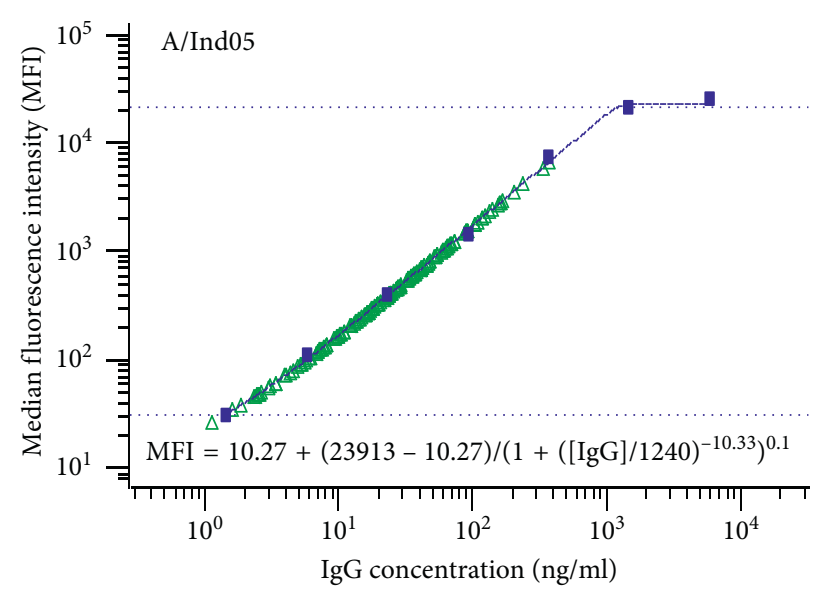

Standard

$\triangle$ Unknown

(b)

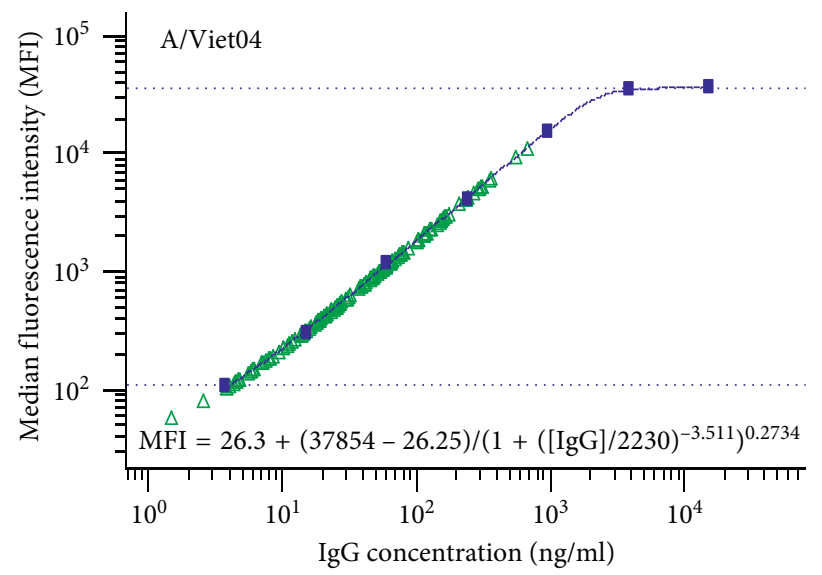

(d)

Figure 3: The examples of standard curve and 5PL fitting formula examples generated by mPlex-Flu assay. (a) The standard curves generated with anti-IgG capture antibodies commonly used in traditional immunoassays through fitting a five-parameter logistic regression model. (b-d) The representative subtype-specific standard curves of influenza HAs from total 45 strains, A/Indonesia/5/2005 (A/Ind05, H5), A/Japan/305/1957 (A/Jap57, H2), and A/Viet Nam/1203/2004 (A/Viet04, H5), generated using five-parameter logistic regression models with different parameters for different subtypes. For each graph, the blue markers and line represent a standard curve, either a single curve for multiple strains (a) or a single strain-specific curve (b-d).

TABLE 2: Example of estimated parameter variations in three HA strain subtypes.

\begin{tabular}{lccccc}
\hline \multirow{2}{*}{ HA strain subtype } & \multicolumn{5}{c}{ Estimated parameters } \\
& $\theta_{1}$ & $\theta_{2}$ & $\theta_{3}$ & $\theta_{4}$ & $\theta_{5}$ \\
\hline A/Indonesia/5/2005 & -10.33 & 10.27 & 23913 & 1240 & 0.1 \\
A/Japan/305/1957 & -11.72 & 14.4 & 17379 & 379.7 & 0.1 \\
A/Viet Nam/1203/2004 & -3.511 & 26.25 & 37854 & 2230 & 0.2734 \\
SEM of estimated & 2.54 & 4.79 & 6038.17 & 534.57 & 0.058 \\
parameters & & & & & \\
\hline
\end{tabular}

$\mathrm{SEM}=$ standard error of the mean.

subtype variations. We noticed that the estimated mean concentrations in the logarithm are different, especially at baseline and end of study measurements. Thus, biases might be introduced to the estimated mean concentrations when strain subtype variations are not taken in account in the data analysis when comparing different treatment groups in clinical vaccine studies. The results shown in Figure 4 suggest that the parameters relating MFI to protein concentration estimated parameters from the five-parameter logistic regression models are different for different $\mathrm{H} 5$ strain subtypes and are also different from the estimated parameters in the common standard curve used for all H5 strain subtypes.

Then, we conducted pairwise comparisons between different treatment groups across different time points within the framework of the linear mixed effects model (Figure 5). Among the 276 pairwise comparisons resulting from the linear mixed effects models conducted in SAS v9.4 (SAS Institute Inc., Cary, NC), 234 pairwise comparisons showed significant differences when the strain subtype variation was taken into account. Meanwhile, 230 out of 276 pairwise comparisons showed significant differences when 


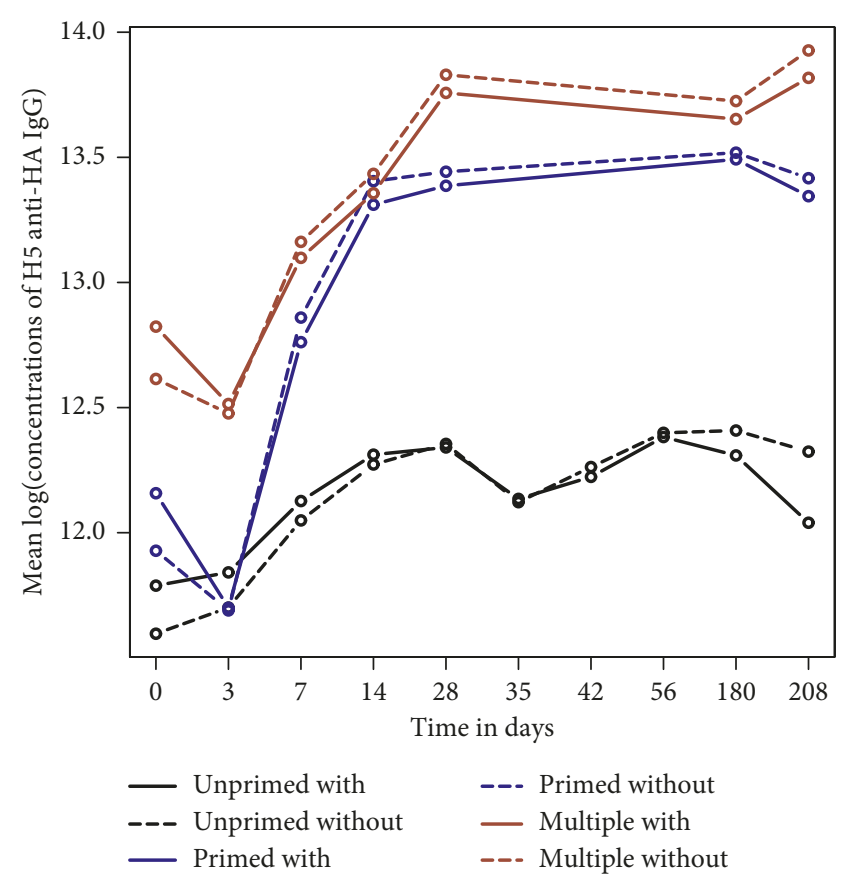

FIGURE 4: The longitudinal estimates from linear mixed effects models with and without considering the H5 strain subtype variations. The mean of longitudinal log concentration showed a total of 21 strains of H5 anti-HA IgG for each of the three different vaccine treatment groups (UPR, PR, and MPR), with and without strain subtype variation taken into account estimated from the linear mixed effects models with adjustment for the differences in dosages, batches, gender, ethnicity, and time points using the restricted maximum likelihood methods.

the strain subtype variation was not taken into account in the data analysis. Although there were only 4 differences in the total number of rejections, there were 22 pairwise comparisons having inconsistent results between the analysis taking the strain subtype variation into account and the analysis not taking the strain subtype variation into account. Table 3 gives some examples of inconsistent results in those pairwise comparisons. It is noticeable from Table 3 that some significant differences might be missed and some significant differences might be false positives if subtype variation is not taken into account in the data analysis. Examples from Table 3 indicate the results of group comparisons in vaccine studies will be affected when strain subtype variation is not taken into account in vaccine data analysis.

3.3. Simulation Study. Monte Carlo simulation studies [23] were conducted to assess the importance of including strain subtype variation in immunoassay when comparing different treatment groups. We suspect that both false positives and false negatives will be inflated if strain subtype variations are not taken into account in vaccine data analysis when comparing different treatment groups, given what we have observed in our analysis of the $\mathrm{H} 5$ vaccine data (Table 3 ). Therefore, the FDR, sensitivity, and specificity were compared between group differential analyses with and without considering strain subtype variations.
3.4. Simulation Description. Using above mPlex-Flu assay data from $\mathrm{H} 5$ clinical trial, we estimated the overall mean concentration from the five-parameter logistic regression model with coefficients estimates of $\widehat{\theta}=(5.44,9.68,0.41$, $4.42,0.81)$, which gave an overall mean concentration of 12.35 in the simulation. The logarithm of IgG antibody reactivity levels $y_{i j k}^{*}$ from $i$ th treatment group, $j$ th influenza virus strain subtype, and $k$ th sample $(i=1,2 ; j=1,2,3$; $k=1,2, \ldots, n)$ was simulated according to the following linear regression models:

$$
y_{i j k}^{*}=\mu_{0}+g_{i}+s_{j}+\epsilon_{i j k}
$$

where $\mu_{0}=12.35, g_{1}$ denotes the difference between the first treatment group and the second treatment group that takes sequential values from 1 to 2 for true difference situation and takes a value of 0 for no difference situation, and $g_{2}$ is set at 0 in the simulation. The strain subtype difference was denoted by $s_{j}$. For simplicity, three strain subtypes were included in the simulation. The parameter $s_{1}$ denoted the difference between strain subtype 1 and strain subtype 3, which follows a random normal distribution with mean $\mu_{s 1}$ and standard deviation of 0.08 . Similarly, $s_{2}$ denotes the difference between strain subtype 2 and strain subtype 3 , which follows a random normal distribution with mean $\mu_{s 2}$ and standard deviation $\sigma=0.1$. The parameter $s_{3}$ is set at 0 in the model.

In the simulations, we used four sets of combinations of $\mu_{s 1}$ and $\mu_{s 2}$ in the simulation studies: (1) $\mu_{s 1}=0.5, \mu_{s 2}=1.0$; (2) $\mu_{s 1}=0.3, \mu_{s 2}=0.6$; (3) $\mu_{s 1}=0.1, \mu_{s 2}=0.3$; and (4) $\mu_{s 1}=0.0, \mu_{s 2}=0.0$. The parameter $\epsilon_{i j k}$ denotes random errors that have independently identical normal distributions with mean $\mu=0$ and variance $\sigma^{2} \mu_{0}^{\tau}$. According to the experimentally measured IgG concentration data, plausible values for $\sigma$ could be $(0.05,0.08,0.1)$ and plausible values for $\tau$ could be sequential values from 0.5 to 1.2 with an interval of 0.1 . We used $\mu_{0}=12.35$ to obtain a mean variance value of 0.06 , i.e., $\operatorname{mean}\left\{\operatorname{var}\left(\epsilon_{i j k}\right)\right\}=\operatorname{mean}\left\{\sigma^{2} \mu_{0}^{\tau}\right\}=0.06$.

We simulated 1,000 random samples of paired data $\left(y_{i j k}^{*}, x_{i j k}\right)$ from the linear regression equation, where $x_{i j k}$ is the design matrix for the linear regression model. Among the 1,000 random samples, the proportion with true differences between the treatment groups was set at $\pi_{1}$ $\left(\pi_{1}=0.25,0.30,0.40,0.50,0.60,0.75,0.90\right)$. When $\pi_{1}=0.25$, there are $1,000 \times \pi_{1}=1,000 \times 0.25=250$ random samples having true differences between the treatment groups. The magnitude of the true differences ranged from 1-2 with an increment of $((2-1) / 250)=0.004$ from the first to the 250 th random sample. The true difference between the treatment groups is 0 for the remaining 750 samples in the simulation. The sample size for each random sample was set at $n=15$ for each of the treatment groups.

Traditional approaches to quantify the IgG antibodies against different influenza HA subtypes in human serum use a common standard curve to estimate the IgG concentration across all strains and subtypes. Such approaches generally test for a statistically significant difference between treatment groups without considering subtype antibody binding variations. The following regression models are commonly used to fit the logarithm of the concentration data: 

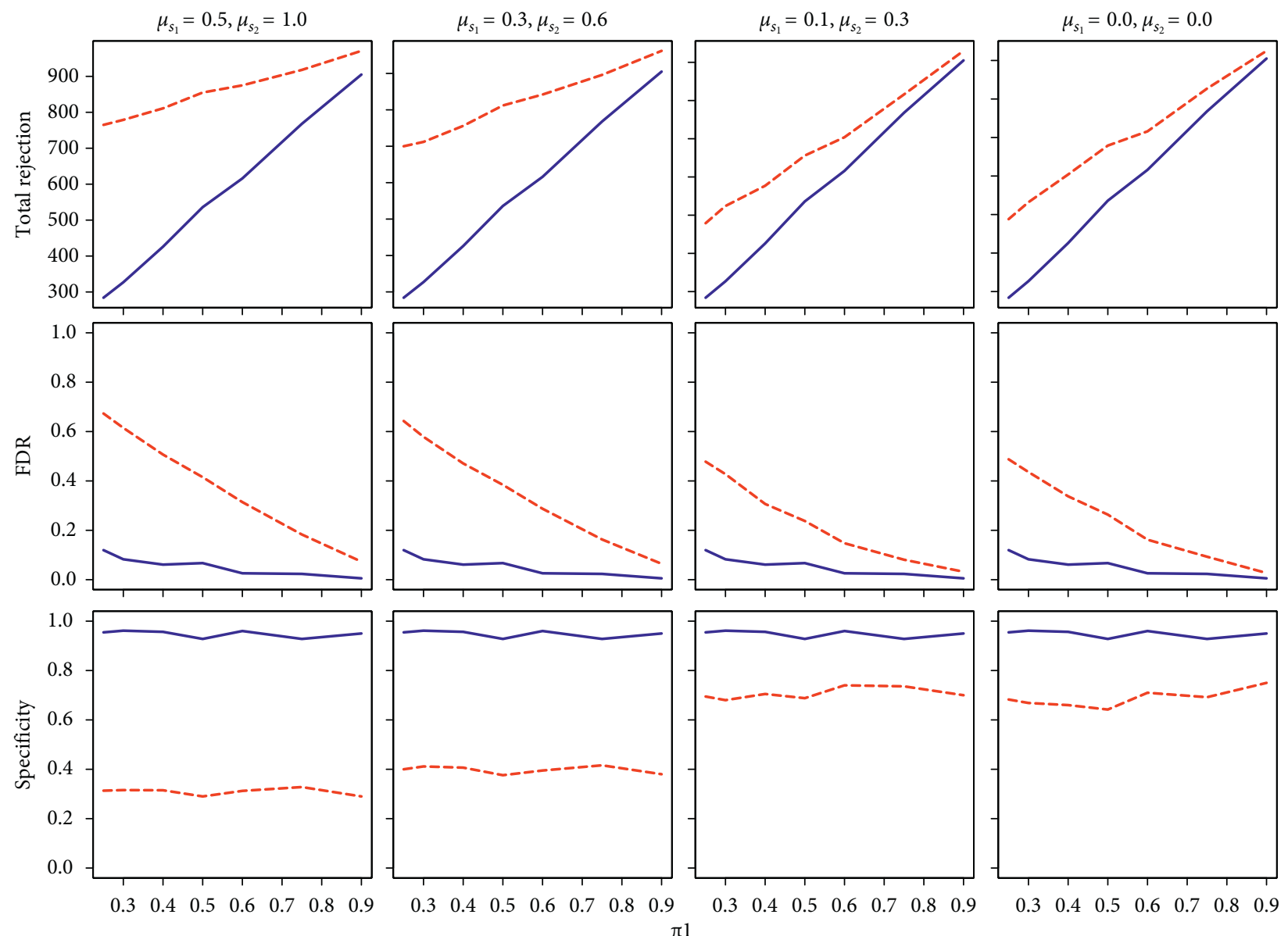

- With subtype variation
--- Without subtype variation

FIGURE 5: Simulation results on total rejection, FDR, sensitivity, and specificity under different simulation settings with sample size of 15 in each group. The FDRs for comparing different groups were markedly smaller when the strain subtype variations were considered in the data analysis, especially when the proportion of true differences between treatment groups $\left(\pi_{1}\right)$ were small. The specificities were much larger when the strain subtype differences were taken into account.

TABLE 3: Examples of inconsistent results from pairwise comparisons with and without consideration of strain subtype variations in the clinical vaccine data analysis.

\begin{tabular}{|c|c|c|c|c|c|c|c|c|c|}
\hline \multirow[t]{2}{*}{ Group } & \multirow[t]{2}{*}{ Time in days } & \multirow[t]{2}{*}{ Group } & \multirow[t]{2}{*}{ Time in days } & \multicolumn{3}{|c|}{$\begin{array}{c}\text { With consideration of subtype } \\
\text { variation }\end{array}$} & \multicolumn{3}{|c|}{$\begin{array}{c}\text { Without consideration of subtype } \\
\text { variation }\end{array}$} \\
\hline & & & & $\Delta$ & SE of $\Delta$ & $P$ value & $\Delta$ & SE of $\Delta$ & $P$ value \\
\hline MPR & 0 & MPR & 3 & 0.3092 & 0.0643 & $<0.0001$ & 0.1376 & 0.0825 & 0.0953 \\
\hline MPR & 180 & PR & 180 & 0.1609 & 0.0956 & 0.0927 & 0.2062 & 0.0938 & 0.0281 \\
\hline PR & 14 & PR & 180 & -0.1812 & 0.0494 & 0.0002 & -0.1139 & 0.0610 & 0.0620 \\
\hline PR & 28 & $\mathrm{PR}$ & 180 & -0.1056 & 0.0453 & 0.0198 & -0.0762 & 0.0583 & 0.1913 \\
\hline UP & 56 & UP & 208 & 0.3424 & 0.0842 & $<0.0001$ & 0.0751 & 0.1031 & 0.4663 \\
\hline UP & 180 & UP & 208 & 0.2689 & 0.0666 & $<0.0001$ & 0.0843 & 0.0854 & 0.3237 \\
\hline
\end{tabular}

$\mathrm{SE}=$ standard error; $\mathrm{PR}=$ primed group; $\mathrm{MPR}=$ multiply primed group; $\mathrm{UP}=$ unprimed group.

$$
y_{i k}=\mu_{0}+g_{i}+\epsilon_{i k}, \quad i=1,2 ; k=1, \ldots, n,
$$

where $y_{i k}$ denotes the logarithm of the concentration data for $i$ th group and $k$ th sample within the $i$ th group, $g_{1}$ denotes the differences between treatment groups and $g_{2}=0 . \epsilon_{i k}$ is assumed to have independent identical distribution of $N\left(0, \sigma^{2}\right)$.
Our approach takes the variation in subtype antibodies and reagents into account. We fit a standard curve for each of the viral HA subtypes using a five-parameter logistic regression model with strain-specific parameters fitted to the logarithm of the concentration data:

$$
y_{i j k}=\mu_{0}+g_{i}+s_{j}+\epsilon_{i j k},
$$


where $y_{i j k}$ denotes the logarithm of the concentration data for $i$ th group, $j$ th strain, and $k$ th sample within the $i$ th group $j$ th strain, $g_{1}$ denotes the differences between treatment groups and $g_{2}=0, s_{1}$ denotes the difference between strain subtypes 1 and $3, s_{2}$ denotes the difference between strain subtypes 2 and 3 , and $s_{3}=0 . \epsilon_{i k}$ is assumed to have independent identical distribution of $N\left(0, \sigma^{2}\right)$. To simplify our simulation studies, we assumed that the correlations among the three subtypes were zero.

\subsection{Simulation Results: Improved FDR Control and Specificity} with Strain Subtype Variation Considered. Figure 5 shows the simulation results comparing the treatment groups with and without strain subtype differences taken into account. We next sought to determine if accounting for influenza HA strain variation would affect the statistical comparison of vaccine response treatment groups from a clinical influenza vaccine study. The data were generated from an mPLEX-flu assay of samples collected during a previous study of responses to an H5 influenza vaccine (DMID 08-0059) [14]. The goal of the trial was to determine if there were significant differences in the anti-HA influenza antibody response between three groups that received intramuscular anti-A/ Indonesia/5/05 H5 influenza vaccine: UPR - no previous exposure to any $\mathrm{H} 5$ vaccine, $\mathrm{PR}-$ had been vaccinated once previously against a different $\mathrm{H} 5$ influenza strain (either A/ Vietnam/1203/04 or a recombinant HA vaccine against A/ Hong Kong/156/97(A/HK97)), and MPR-received two sequential vaccinations against the A/Indonesia/5/05 H5 influenza virus.

The FDRs for comparisons between different treatment groups were markedly smaller and the specificity much greater, when the strain subtype differences were taken into account. When differences in strain subtype were not accounted for, the probability of finding differences between treatment groups was much higher with more significant differences identified between the treatment groups. In contrast, considering strain subtype variation did not affect the sensitivity of statistical comparison between different treatment groups.

We also observed a noticeable decrease in FDRs as the proportion of true differences between the treatment groups increased. In contrast, the differences in specificities did not change even as the proportion of true differences between the treatment groups increased. When the variation in influenza HA subtype differences decreased, we found a concomitant decrease in FDRs and an increase in specificity (Table 4). Even when the mean concentration difference between strain subtypes was zero, the FDRs were still much larger and the specificities were still much smaller when comparing differences between the treatment groups. The inflated FDRs appeared largely due to ignoring strain subtype variations and increased as the proportion of true differences between the treatment groups became smaller.

When the sample size in the simulation studies increased to 30,60 , and 120 in each treatment group, we obtained similar results for the number of total rejections, FDR, sensitivity, and specificity (Supplementary Figures 1-3).
TABLE 4: FDR and specificity comparison with and without considering the strain subtype variations in the treatment groups comparisons at different settings.

\begin{tabular}{|c|c|c|c|c|c|}
\hline Parameter settings & $\pi_{1}$ & \multicolumn{2}{|c|}{$\begin{array}{c}\text { With Without } \\
\text { FDR }\end{array}$} & \multicolumn{2}{|c|}{$\begin{array}{l}\text { With Without } \\
\text { Specificity }\end{array}$} \\
\hline & 0.25 & 0.1197 & 0.6732 & 0.9547 & 0.3133 \\
\hline & 0.30 & 0.0826 & 0.6149 & 0.9614 & 0.3157 \\
\hline Setting 1 & 0.40 & 0.0610 & 0.5068 & 0.9567 & 0.3150 \\
\hline$\mu_{s 1}=0.5$ & 0.50 & 0.0672 & 0.4152 & 0.9280 & 0.2900 \\
\hline \multirow[t]{5}{*}{$\mu_{s 2}=1.0$} & 0.60 & 0.0260 & 0.3143 & 0.9600 & 0.3125 \\
\hline & 0.75 & 0.0234 & 0.1830 & 0.9280 & 0.3280 \\
\hline & 0.90 & 0.0055 & 0.0731 & 0.9500 & 0.2900 \\
\hline & 0.40 & 0.0610 & 0.4709 & 0.9567 & 0.4067 \\
\hline & 0.50 & 0.0672 & 0.3842 & 0.9280 & 0.3760 \\
\hline Setting 2 & 0.60 & 0.0260 & 0.2874 & 0.9600 & 0.3950 \\
\hline$\mu_{s 1}=0.3$ & 0.75 & 0.0234 & 0.1629 & 0.9280 & 0.4160 \\
\hline \multirow[t]{5}{*}{$\mu_{s 2}=0.6$} & 0.90 & 0.0055 & 0.0644 & 0.9500 & 0.3800 \\
\hline & 0.25 & 0.1197 & 0.4781 & 0.9547 & 0.6947 \\
\hline & 0.30 & 0.0826 & 0.4275 & 0.9614 & 0.6800 \\
\hline & 0.40 & 0.0610 & 0.3068 & 0.9567 & 0.7050 \\
\hline & 0.50 & 0.0672 & 0.2378 & 0.9280 & 0.6880 \\
\hline Setting 3 & 0.60 & 0.0260 & 0.1477 & 0.9600 & 0.7400 \\
\hline$\mu_{s 2}=0.1$ & 0.75 & 0.0234 & 0.0809 & 0.9280 & 0.7360 \\
\hline \multirow{4}{*}{$\mu_{s 2}=0.3$} & 0.90 & 0.0055 & 0.0323 & 0.9500 & 0.7000 \\
\hline & 0.25 & 0.1197 & 0.4877 & 0.9547 & 0.6827 \\
\hline & 0.30 & 0.0826 & 0.4361 & 0.9614 & 0.6686 \\
\hline & 0.40 & 0.0610 & 0.3377 & 0.9567 & 0.6600 \\
\hline Setting 4 & 0.50 & 0.0672 & 0.2636 & 0.9280 & 0.6420 \\
\hline$\mu_{s 1}=0.0$ & 0.60 & 0.0260 & 0.1620 & 0.9600 & 0.7100 \\
\hline \multirow{2}{*}{$\mu_{s 2}=0.0$} & 0.75 & 0.0234 & 0.0931 & 0.9280 & 0.6920 \\
\hline & 0.90 & 0.0055 & 0.0270 & 0.9500 & 0.7500 \\
\hline
\end{tabular}

$\mathrm{FDR}=$ false discovery rate.

Thus, only the results from sample size of 15 in each treatment group are presented in Figure 5.

\section{Discussion}

Every year, the WHO selects influenza vaccine strains trying to pick the best influenza virus strains that would be able to represent the circulating virus strains in same or similar antigenicity of HA protein, on the surface of virus. Furthermore, the traditional way for determination of the antigenicity of one influenza virus is to let this virus isolation to react against a panel of ferret antisera, and each antiserum is generated from naive ferret after the infection of one singlespecific influenza virus [2]. However, antigenic data or immunological patterns in human sera are more complicated and difficult to interpret due to exposure histories and cross-reactivity between influenza virus strains [1]. In addition, some important studies showed that early lifetime exposure of influenza virus (imprinting) might provide cross-protection against infection of $\mathrm{H} 5, \mathrm{H} 7$ novel subtype influenza viruses [24]. It is essential to develop an efficient and high throughput assay for the evaluation of those crossreactive antibodies. This novel technique, mPlex-Flu assay, allows us be able to quickly and accurately estimate the humoral immune response after influenza infection or vaccination and rapidly characterize comprehensive 
individual- and population-level heterosubtypic immunity of a broad range of influenza strains.

However, how to quantify the antibodies and their crossreactivities against influenza virus is always a challenge in influenza vaccine studies. The mFlex-Flu assay couples beads with analyte-specific rHAs (antigens) to detect the specific antibodies binding influenza strain-specific HAs. To quantify the amount of active HA-specific antibodies, mPlex-Flu assay assesses both the amount and affinity of antibodies against influenza viral HAs at the same time. Furthermore, there are many modest differences between the replication and infection of subtypes of influenza viruses [25] and the diversity of HA structure and characters between individual subtypes of influenza viruses [26]. In addition, the slight differences in batch, time, and other experiment conditions can also introduce variation from assay to assay. Traditional assays to evaluate specific antibodies against influenza subtype virus or HA, such as HAI [27], MN [28], and ELISA [29], are semiquantitative. They use the highest dilution or endpoint of dilution of serum to determine the titer of the antibodies. Those discrete-ranked readouts of one of 8-14 titer values could introduce imprecision and increase false discoveries. The major problem is that they are not able to provide a precise evaluation normalized by the difference in strains of influenza virus. This presents a big challenge for directly comparing the anti-HA IgG levels against different influenza viruses, within or between subtype of influenza virus, when studying the frequency and binding of crossreactive antibodies against multiple influenza strains.

In order to generate precise continuous values of antibody levels, which rely on the appropriate standard sample to generate a good standard curve, initially, we set up an ELISA assay with the anti-human IgG antibody standard curve, as described previously $[11,13]$. Although using one standard curve can adjust for variation caused by experiment conditions, we still could not determine the types of imprecisions introduced by the variations of deferent strains HAs of influenza virus. In our previous publication $[3,4]$, we introduced a novel multiplex method to quantitatively measure the concentration of rHA-specific antibodies by using the standard reference serum (STD02), similar to SDT01 [3], which is a mixture serum from four subjects having high titer antibodies against seasonal influenza viruses, based on a study of the estimation of weight-based antibody unite, published by Dr. Quataert [12]. Importantly, we also set up independent standard curves for each analyte (influenza virus HA strain) to convert the MFI units of mPlex-Flu assay into concentration-based antibody units.

Within the mPLEX-Flu assay, the HA from various strains do not directly interact. The one class of interactions that could influence the assay is that of competition for antibodies that bind to regions of different HA strains having the same antigenic sequence (epitopes). In our experimental setting, we use excess serum (with antibodies to multiple antigens) or monocloncal antibodies (bind to a single antigenic site) to minimize or eliminate the binding competition between influenza virus strains. Under these conditions, the binding of anti-HA IgG to one HA variation does not affect binding of another HA variation given there are more than enough antibodies available. Similarly, the standard curve of each subtype antibody concentration can be generated to obtain the antibody concentration for each HA subtype. Thus, the binding of each HA variation with antibodies can be treated as independent binding. We are aware that these conditions may not hold true at much lower antibody concentrations but have found such concentrations to be below the usual range for serum antibody. However, it is important to note that the independence assumption gives us more conservative results for our statistical evaluation than dependent assumption. Thus, the simulation results we obtained using the independent assumption are still valid for dependent situation.

The results of this study showed significant variations in IgG-rHA binding model parameter estimates among different rHA strains. This finding is likely due to differences in reagent surface density and staeric hinderence between subtypic recombinant HA proteins (rHAs). When different standard curves were used for each strain subtype, the concentration differences between different strain subtypes could be taken into account when comparing different treatment groups. When one common standard curve was used for all strain subtypes, the concentration differences between different strain subtypes were embedded in the random errors.

Our simulation studies have shown that without considering the variation in strain subtypes, the Type I error associated with testing differences between treatment groups will be inflated and the specificity will be lowered, compared to analysis with the strain subtype variation taken into account. Our case studies also showed inconsistent results in pairwise group comparisons when we took or did not take the variations in strain subtypes into account. Therefore, the estimated differences of interested group comparisons are less biased if the strain subtype variations are taken into account in the data analysis by estimating concentrations from the individual standard curves of each strain subtype. Meanwhile, the type I error of testing interested group differences will be reduced and the specificity will also be increased. Thus, we recommend taking strain subtype variations into account in clinical vaccine research.

This study provides solid statistic evidence to support our published method to quantify the concentration unit of antibodies in mPlex-Flu assay. It also suggests that it is more accurate to directly compare the concentration units between subtype analytes after adjustment by each individual standard curve.

\section{Data Availability}

The data used to support the findings of this study are available from the corresponding author upon request.

\section{Disclosure}

The content is solely the responsibility of the authors and does not necessarily represent the official views of the $\mathrm{Na}$ tional Institutes of Health. None of the above funders had any role in study design, data collection and analysis, decision to publish, or preparation of the manuscript. 


\section{Conflicts of Interest}

The authors declare that there are no conflicts of interest.

\section{Authors' Contributions}

DL, JW, and MZ conceived and designed the study. JT directed the influenza vaccine clinical trial (DMID 08-0059) and provided samples. DL conducted simulation studies and analyzed the clinical trial data. DL, JW, and MZ wrote and edited the manuscript. All the authors approved the final version of the manuscript. DL and JW equally contributed to this work.

\section{Acknowledgments}

The authors would like to thank Shannon Hilchey for critical reading of the manuscript and Judy Grastorf for her excellent editorial assistance. The authors would like to thank Enago (http://www.enago.com) for the English language review. This work was supported by grants from the National Institutes of Health, National Institute of Allergy and Infectious Diseases, including AI098112 and AI069351 (MZ, JW, and JG) and R21AI138500 (MZ, JW, and JG). The project described in this publication was also supported by the University of Rochester Clinical and Translational Science Award UL1 TR002001 from the National Center for Advancing Translational Sciences of the National Institutes of Health (DL, MZ, JW, and JG).

\section{Supplementary Materials}

Supplementary Figures 1-3: simulation study results with total rejections, FDR, sensitivity, and specificity when the sample size is 30,60 , or 120 in each treatment group. (Supplementary Materials)

\section{References}

[1] D. J. Smith, A. S. Lapedes, J. C. de Jong et al., "Mapping the antigenic and genetic evolution of influenza virus," Science, vol. 305, no. 5682, pp. 371-376, 2004.

[2] I. G. Barr, J. McCauley, N. Cox et al., "Epidemiological, antigenic and genetic characteristics of seasonal influenza $\mathrm{A}(\mathrm{H} 1 \mathrm{~N} 1), \mathrm{A}(\mathrm{H} 3 \mathrm{~N} 2)$ and $\mathrm{B}$ influenza viruses: basis for the WHO recommendation on the composition of influenza vaccines for use in the 2009-2010 Northern Hemisphere season," Vaccine, vol. 28, no. 5, pp. 1156-1167, 2010.

[3] J. Wang, S. P. Hilchey, O. Hyrien et al., "Multi-dimensional measurement of antibody-mediated heterosubtypic immunity to influenza," PLoS One, vol. 10, no. 6, Article ID e0129858, 2015.

[4] J. Wang, S. P. Hilchey, M. DeDiego et al., "Broad crossreactive igg responses elicited by adjuvanted vaccination with recombinant influenza hemagglutinin ( $\mathrm{rHA}$ ) in ferrets and mice," PLoS One, vol. 13, no. 4, Article ID e0193680, 2018.

[5] T. Horimoto and Y. Kawaoka, "Influenza: lessons from past pandemics, warnings from current incidents," Nature Reviews Microbiology, vol. 3, no. 8, pp. 591-600, 2005.

[6] G. K. Hirst, "The quantitative determination of influenza virus and antibodies by means of red cell agglutination," Journal of Experimental Medicine, vol. 75, no. 1, pp. 49-64, 1942.
[7] G. K. Hirst, "Adsorption of influenza hemagglutinins and virus by red blood cells," Journal of Experimental Medicine, vol. 76, no. 2, pp. 195-209, 1942.

[8] A. L. Frank, J. Puck, B. J. Hughes, and T. R. Cate, "Microneutralization test for influenza $A$ and $B$ and parainfluenza 1 and 2 viruses that uses continuous cell lines and fresh serum enhancement," Journal of Clinical Microbiology, vol. 12, no. 3, pp. 426-432, 1980.

[9] E. J. Remarque, I. A. de Bruijn, W. J. A. Boersma, N. Masurel, and G. J. Ligthart, "Altered antibody response to influenza $\mathrm{H} 1 \mathrm{~N} 1$ vaccine in healthy elderly people as determined by $\mathrm{HI}$, ELISA, and neutralization assay," Journal of Medical Virology, vol. 55, no. 1, pp. 82-87, 1998.

[10] M. S. Zand, J. Wang, and S. Hilchey, "Graphical representation of proximity measures for multidimensional data: classical and metric multidimensional scaling," Mathematica Journal, vol. 17, 2015.

[11] A. D. Henn, S. Wu, X. Qiu et al., "High-resolution temporal response patterns to influenza vaccine reveal a distinct human plasma cell gene signature," Scientific Reports, vol. 3, no. 1, p. 2327, 2013.

[12] S. A. Quataert, K. Rittenhouse-Olson, C. S. Kirch et al., "Assignment of weight-based antibody units for 13 serotypes to a human antipneumococcal standard reference serum, lot 89-s(f)," Clinical and Vaccine Immunology, vol. 11, no. 6, pp. 1064-1069, 2004.

[13] A. D. Henn, M. Laski, H. Yang et al., "Functionally distinct subpopulations of CpG-activated memory B cells," Scientific Reports, vol. 2, no. 1, p. 345, 2012.

[14] J. L. Nayak, K. A. Richards, H. Yang, J. J. Treanor, and A. J. Sant, "Effect of influenza $\mathrm{A}(\mathrm{H} 5 \mathrm{~N} 1)$ vaccine prepandemic priming on $\mathrm{CD}^{+}$T-cell responses," Journal of Infectious Diseases, vol. 211, no. 9, pp. 1408-1417, 2015.

[15] B. T. West, K. B. Welch, and A. T. Galecki, Linear Mixed Models: A Practical Guide using Statistical Software, Chapman \& Hall/CRC, New York, NY, USA, 2007.

[16] M. J. R. Healy, "Statistical analysis of radioimmunoassay data," Biochemical Journal, vol. 130, no. 1, pp. 207-210, 1972.

[17] R. A. Dudley, P. Edwards, R. P. Ekins et al., "Guidelines for immunoassay data processing," Clinical Chemistry, vol. 31, no. 8, pp. 1264-71, 1985.

[18] P. G. Gottschalk and J. R. Dunn, "The five-parameter logistic: a characterization and comparison with the four-parameter logistic," Analytical Biochemistry, vol. 343, no. 1, pp. 54-65, 2005.

[19] J. J. Grady and R. W. Helms, "Model selection techniques for the covariance matrix for incomplete longitudinal data," Statistics in Medicine, vol. 14, no. 13, pp. 1397-1416, 1995.

[20] R. A. Mclean, W. L. Sanders, and W. W. Stroup, "A unified approach to mixed linear models," American Statistician, vol. 45, no. 1, pp. 54-64, 1991.

[21] P. M. Bentler and J. Liang, "A unified approach to two-level structural equation models and linear mixed effects models," Random Effect and Latent Variable Model Selection, vol. 192, pp. 95-119, 2008.

[22] M. G. Kenward and J. H. Roger, "Small sample inference for fixed effects from restricted maximum likelihood," Biometrics, vol. 53, no. 3, pp. 983-997, 1997.

[23] W. K. Hastings, "Monte Carlo sampling methods using Markov chains and their applications," Biometrika, vol. 57, no. 1, pp. 97-109, 1970.

[24] Y. Qin, P. W. Horby, T. K. Tsang et al., "Differences in the epidemiology of human cases of avian influenza A(H7N9) and $\mathrm{A}(\mathrm{H} 5 \mathrm{~N} 1)$ viruses infection," Clinical Infectious Diseases, vol. 61, no. 4, pp. 563-571, 2015. 
[25] L. A. Santos, S. Solá, C. M. Rodrigues, and H. Rebelo-deAndrade, "Distinct kinetics and pathways of apoptosis in influenza A and B virus infection," Virus Research, vol. 205, pp. 33-40, 2015.

[26] D. C. Ekiert, G. Bhabha, M.-A. Elsliger et al., "Antibody recognition of a highly conserved influenza virus epitope," Science, vol. 324, no. 5924, pp. 246-251, 2009.

[27] G. Wilson, Z. Ye, H. Xie, S. Vahl, E. Dawson, and K. Rowlen, "Automated interpretation of influenza hemagglutination inhibition (HAI) assays: is plate tilting necessary?," PLoS One, vol. 12, no. 6, Article ID e0179939, 2017.

[28] Y. Lin, Y. Gu, and J. W. McCauley, "Optimization of a quantitative micro-neutralization assay," Journal of Visualized Experiments, no. 118, 2016.

[29] R. Nachbagauer, A. Choi, A. Hirsh et al., "Defining the antibody cross-reactome directed against the influenza virus surface glycoproteins," Nature Immunology, vol. 18, no. 4, pp. 464-473, 2017. 


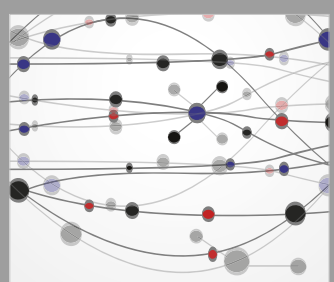

The Scientific World Journal
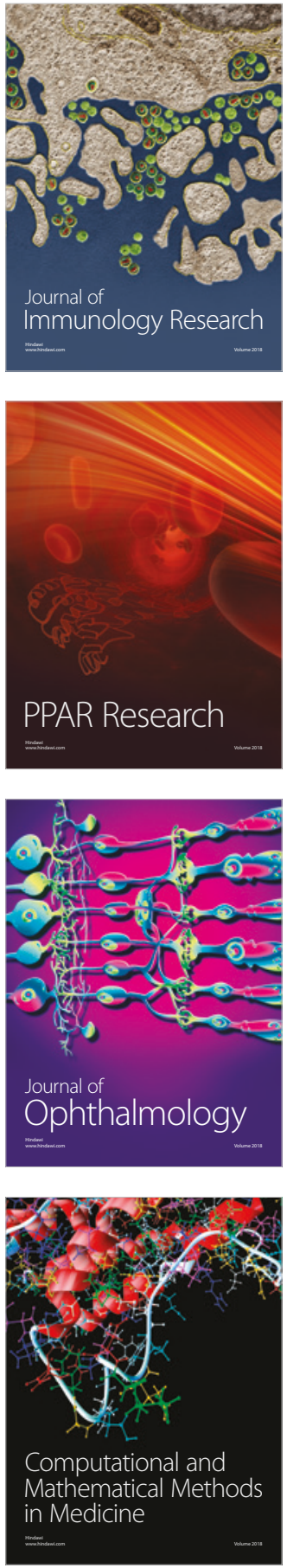

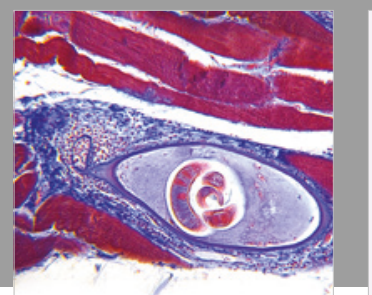

Gastroenterology Research and Practice

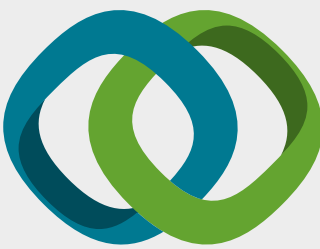

\section{Hindawi}

Submit your manuscripts at

www.hindawi.com
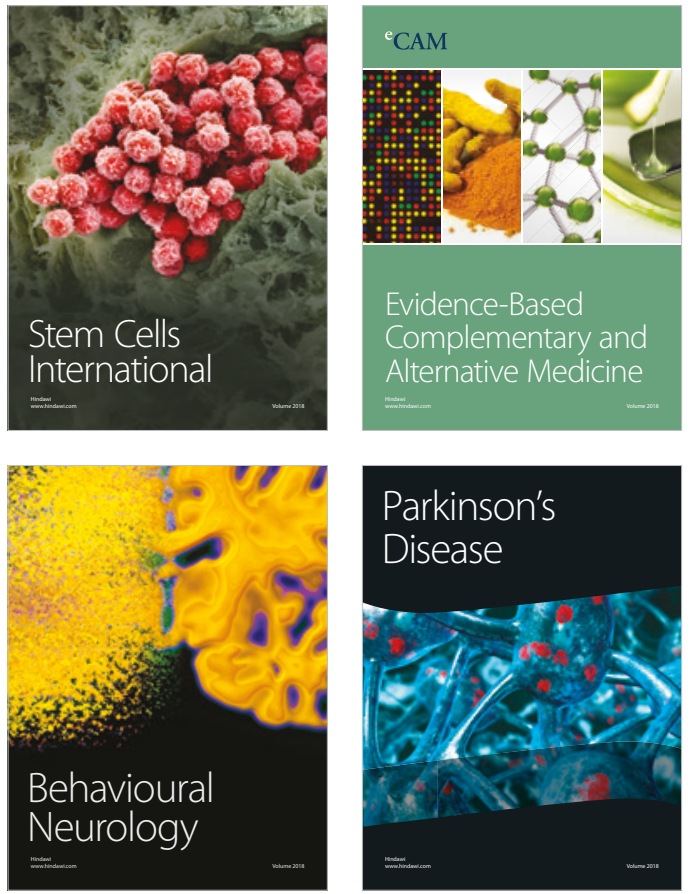

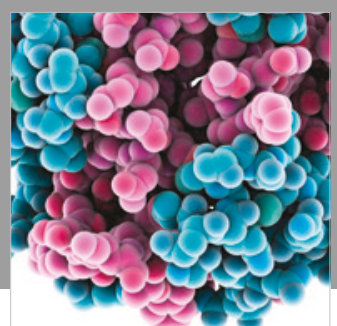

ournal of

Diabetes Research

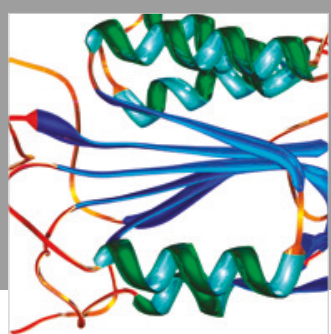

Disease Markers
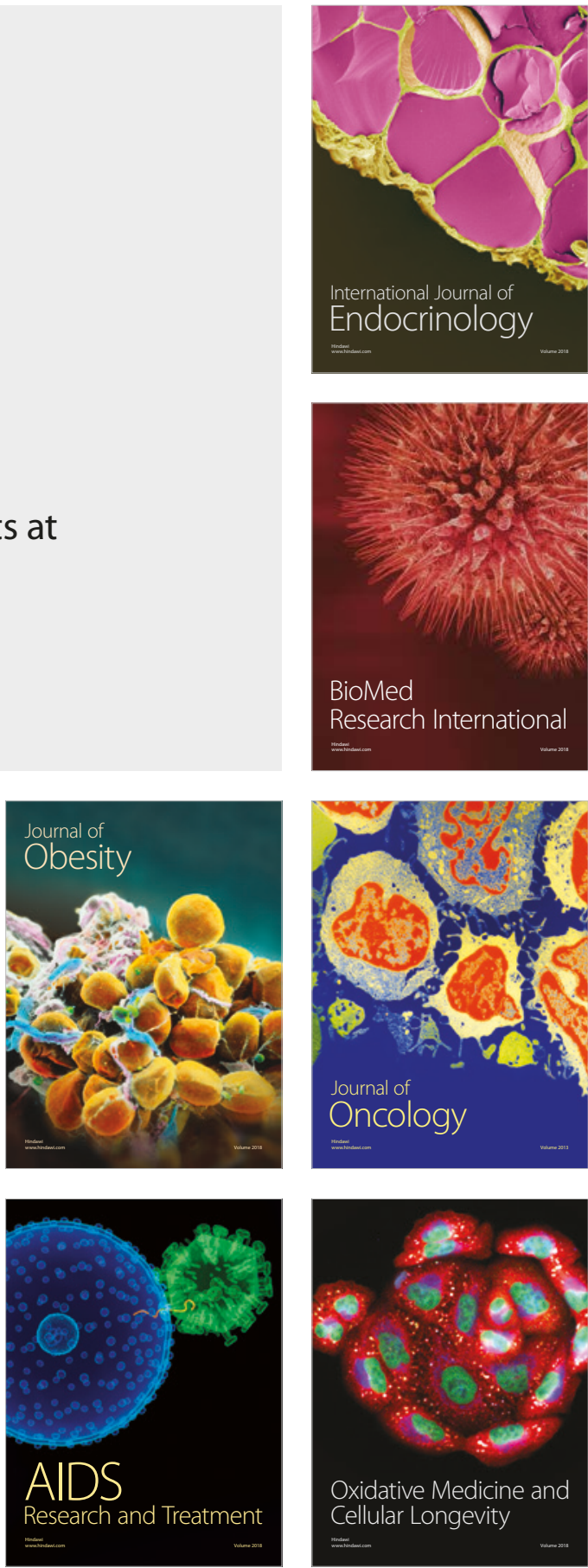